\title{
Perspective postcoloniale, écriture francophone et réponse critique à la crise de la modernité proto-anthropocène
}

\section{Laté LAWSON-HELLU, Université Western (Canada)}

\section{La problématique}

Il s'agit d'une réflexion épistémologique, et donc «théorique », qui repose sur deux travaux proposés dans le cadre de la formalisation de la perspective herméneutique postcoloniale et dans le cadre de la conceptualisation de l'Anthropocène. Je ne reprends pas ici l'immense travail de conceptualisation, de reformulation, voire de contestation de l'approche postcoloniale. Le cadre de la réflexion ne s'y prête pas. Cet immense travail informe cependant les « résultats » qui en sont considérés, telle la question de la résistance, dans la présente réflexion. Pour la question de l'Anthropocène, par contre, le débat est en cours dont nous retenons un des aspects, celui de sa circonscription historique et herméneutique, épistémologique donc, pour le présent travail d'exploration des voies de rencontre de la théorie postcoloniale et des soucis écologiques actuels dont rend compte le paradigme de l'Anthropocène. Je propose de considérer ce dernier comme la crise de la modernité, du point de vue épistémologique des conditions de vie de l'être humain sur la planète. Le premier travail porte sur la question du nativisme, ou du retour au monde prémoderne de la tradition, dans le cadre de la critique de la modernité coloniale. C'est une telle critique de la modernité coloniale que la perspective postcoloniale cherche à lire dans le corpus « postcolonial », c'est-à-dire les œuvres littéraires ou artistiques produites dans le cadre historique et épistémologique du colonialisme européen. Il est entendu que ces œuvres se positionnent face à ce fait colonial dans leur antinomie à son fondement idéologique et hégémonique. Le principe de la résistance traduit cette posture qui devient ontologique, c'est-à-dire qui renvoie à ce qui fait la particularité matérielle, "physique», de l'être humain, et sa particularité non-matérielle, « métaphysique », si l'on veut. La propension de l'être humain au principe métaphysique, au cœur notamment de sa capacité à penser un au-delà de la réalité qu'il met alors dans le fait religieux, est un exemple de ce principe métaphysique. La catégorie du nativisme est proposée dans la réflexion épistémologique de la théorie postcoloniale pour en récuser la «tentation», au nom de l'intégration progressive du fait colonial même dans le discours et la conception de l'espace que prennent en charge les corpus postcoloniaux. Il est entendu de même que le corpus postcolonial 
ne précède pas le fait colonial et donc s'inscrit dans un espace et un lieu dont la reconstitution ne peut qu'intégrer cette réalité coloniale, ou le legs du fait colonial. Je voudrai prendre à contre-pied ce postulat, dans la mesure de l'échec que démontre le principe de la modernité aujourd'hui, tel que cherche à le traduire le paradigme de l'Anthropocène.

Par ce paradigme, en effet, il faut penser le résultat négatif de l'action humaine sur la planète depuis l'émergence de la modernité au seizième siècle et ses développements ultérieurs. Les effets de ce développement se ressentent dans tous les domaines d'activité et d'existence de l'être humain, au point de menacer sa propre survie sur la planète, mais aussi de nécessiter la recherche de solutions à l'état de sursis créé. Celui-ci s'est exacerbé notamment en ce début du vingt-et-unième siècle, ne serait-ce que par les changements climatiques observables sur l'ensemble de la planète, ou, plus récemment, par la crise sanitaire aggravée par les moyens de communication, de «déplacement », amplifiés par le progrès de la modernité. C'est dans le cadre de cette nécessité de recherche de solutions à la modernité que la récusation du nativisme perd de sa pertinence pour l'objectif éthique au cœur de la pensée postcoloniale.

Pour cette pensée postcoloniale, le principe éthique demeure cette recherche, dans l'expression artistique, d'une alternative à l'ordre colonial défini au prime abord par le fait hégémonique. Pour le sujet colonisé et son expression artistique, il s'agit en effet, et avant tout, suivant les postulats de la théorie postcoloniale, de s'opposer au principe de la tabula rasa propre au fait colonial, et de sauvegarder, ou de rappeler la nécessité de sauvegarder, les faits de cultures, d'existence, de pensée ontologique que cherche à infirmer le fait colonial dans son déploiement discursif et idéologique. Le principe du nativisme redevient, dans la lecture des œuvres du corpus colonial, cette alternative qui coïncide avec la recherche de voies de sortie de la crise de la modernité-Anthropocène. Pour le cadre précis de cet article, je vais présenter brièvement la réflexion postcoloniale sur le nativisme chez Benita Parry (1994), et la réflexion sur la recherche de solution, dans la perspective de l'Anthropocène, chez Barbara Glowczewski et Christophe Laurens (2018). L'hypothèse au cœur de la réflexion voudrait que la perspective postcoloniale, dans sa portée herméneutique et éthique, permette de formuler, dans les œuvres du corpus postcolonial, les alternatives épistémologiques à la crise de l'Anthropocène.

La propre assise critique de la réflexion réside dans les propositions éthiques de la perspective écolinguistique (Stibbe 2015) qui demande de rechercher dans les usages de la langue ceux qui sont favorables à la vie, et de les promouvoir, et de rechercher ceux de ces usages qui 
sont antinomiques à la vie, et de les dénoncer. Son postulat est que c'est par les usages de la langue que nous donnons sens à notre vie et à notre existence en collectivité. De dire que la perspective postcoloniale, par le biais du principe nativiste réévalué, devient une modalité de recherche de solution à la crise existentielle de l'être humain, du fait de la modernité, par le biais du fait colonial européen, participe de ces postulats herméneutiques de l'écolinguistique. Autrement dit, la perspective postcoloniale, en tant que cadre herméneutique, critique, dans son antinomie au fait colonial, participe de ces usages de la langue qui sont favorables à la vie et qu'il faut promouvoir.

\section{La question du nativisme}

Dans la réflexion postcoloniale des premières heures, s'est en effet posée la question de la construction de la subjectivité du sujet colonisé, dans sa lutte ou résistance contre l'hégémonie coloniale. En tant qu'objet de discours, l'identité, ou la subjectivité, ainsi construite court le risque, dans sa dimension a priori discursive, de reconduite des catégories imagologiques du discours colonial dans la recherche de base ontologique de construction de cette subjectivité. Pour Parry, qui participait à ce débat, il existe certes des bases concrètes, historiques et non-verbales de telles bases précoloniales pouvant servir au processus de construction de la subjectivité non-coloniale. Cependant, aussi, les tentatives anticoloniales de distanciation des catégories construites par le discours colonial contre le colonisé courent ce risque de se construire à partir de telles catégories.

If we look at the work of contemporary critics recuperating figures of colonial resistance, not from the rhetorical strategies of the dominant discourses but by revisiting dispersed and connotative informal sources, these projects do not appear as preoccupied with victimage, or as enacting a regressive search for an aboriginal and intact condition/tradition from which a proper sense of historicity is occluded - charges which have been made against such undertakings. As an instance of a resistance mode available to the colonial Caribbean, Wilson Harris cites limbo dancing, a practice stemming from Africa and reinterpreted on the slave ships of the Middle Passage, and which although indebted to the past - as is voodoo - is not an imitation of that past but rather « a crucial inner re-creative response to the violations of slavery and indenture and conquest ». (Harris 14 ; Parry 173)

Parry évoque dans ce sens l'exemple de l'identité hindoue, dans le contexte colonial et postcolonial indien, où la volonté de fonder l'origine de cette identité dans les temps védiques entraîne un moindre intérêt pour l'identité collective laïque qui participe de la question identitaire nationale dans ce contexte post-colonial indien. Une telle question, en soi, est au cœur de la réflexion actuelle sur les modalités de définition ou de conception de l'identité nationale en Inde. Pour Parry, 
cependant, cette tentation du «nativisme » est ce que la réflexion épistémologique postcoloniale doit récuser ou éviter. Autrement dit, dans ses postulats, la perspective théorique elle-même se donne des limites dans ses modes de lecture du texte postcolonial. Pour Parry, même Édouard Glissant aurait symboliquement proposé non de regarder l'arbre Acoma du passé disparu, mais de s'en remémorer uniquement devant la mosaïque identitaire qu'était devenu le contexte identitaire caribéen :

A very different impulse towards recuperating a very different history marked by discontinuities and erasures is attested by Edouard Glissant whose repeated references to the Acoma tree intimates that the need to renew or activate memories is distinct from the uncritical attempt to conserve tradition : «One of the trees that has disappeared from the Martinican forest. We should not get too attached to the tree, we might then forget the forest. But we should remember it ». (Glissant 260 ; Parry 174)

Une telle position prend pour acquis que l'histoire coloniale aura généré dans l'espace colonisé puis post-colonial un nouveau rapport à la réalité où les résultats de cette histoire font désormais partie du fait identitaire à construire. Dans l'actualité de la crise de l'Anthropocène, une telle perception de la réalité de l'espace colonisé devient problématique. La question de la modernité sous-jacente à une telle position est devenue le problème, non seulement pour l'espace anciennement colonisé, mais pour l'espace de l'ancien colonisateur. Il en résulte une question cruciale, celle du maintien de ce legs-problème de l'histoire coloniale, c'est-à-dire de la modernité. S'il faut chercher désormais des solutions au problème créé sur les conditions de vie sur la planète, par le fait de la modernité ou, pour nous, ici, le fait colonial, le discours nativiste que la perspective postcoloniale récusait à ses débuts demande une révision nécessaire près d'un demi-siècle après son émergence.

Pour Parry, la tentative de mythologisation du passé précolonial est une source de création de subjectivité dans les communautés marginalisées. Cette tentative ne devrait cependant pas occulter sa dimension de «représentation», et donc d'inefficacité dans la lutte pour la reconnaissance du sujet colonisé et la récupération de son agentivité face au discours colonial : «There are moreover critics who testify to the possibility that the identity struggle of one community can serve as a model for other resistant discourses, since the self-definition articulated by, say, the black or the Jew in defiance of received representations can be communicated to different situations of contest against the authority of the dominant by marginals, exiles and subjugated populations (see Diawara (1991) and Grosz (1990)) » (176). Pour elle, il ne s'agirait 
plus que d'une « fiction d'identité », lorsqu'elle évoque le contexte diasporique de la construction d'identité comme contrepoids au discours jugé monolithique devant une réalité qui serait désormais plurielle et polysémique. Cela l'amène à sa réflexion sur le nativisme qu'elle considère comme pertinent jusqu'à un certain point. Elle lui appose néanmoins le double processus de la contre-identification et de la dé-identification pour souligner dans quelle mesure le discours oppositionnel du sujet colonisé peut être dans une re-production des propres catégories qu'il cherche à invalider, ou dans un « parasitage » par le discours dominant (Parry 176-177).

Il ressort de cette réflexion une tentative de distanciation de la construction identitaire du sujet colonisé d'avec le modèle nationaliste du champ politique. On reconnaîtra ici la critique de ces premières formulations épistémologiques des champs d'intérêt de la pensée postcoloniale. Cette peur, en somme, ne peut désormais se formuler devant une crise générée par l'histoire des cinq siècles de la modernité, et qui excède le cadre politique du nationalisme opposé au cosmopolitisme. Dans un discours populaire de l'actualité sanitaire de ces débuts des années 2020, il est aussi indiqué que la pandémie est résolument « mondiale ». Pour la perspective postcoloniale, la pertinence qu'elle dérive, depuis son émergence, de la mise au jour, et donc de l'infirmation, des catégories idéologiques de fonctionnement du système colonial, ou du système hégémonique occidental, devient également sa pertinence pour penser l'actualité de la modernité conceptualisée, dans sa crise planétaire, sous le paradigme de l'Anthropocène, ou encore, de l'Occidentalocène, ou, ailleurs, du Capitalocène. Cette pertinence de la perspective postcoloniale passe donc par sa reformulation de l'intérêt ontologique de ce qui était désigné sous le paradigme du nativisme, et rejeté à ce titre. La solution à la crise de l'Anthropocène ou de la modernité passe par une sortie de cette modernité et un retour à la Terre, à la Nature, comme le propose la romancière kanak de la Nouvelle-Calédonie, Déwé Gorodé.

Pour Gorodé, en effet, l'avenir de son pays, dans sa lutte pour l'indépendance d'avec la France, passe par la reconnaissance des marqueurs de la tradition, de la « coutume», comme cela se dit dans un espace culturel en processus de construction identitaire post-coloniale, justement. C'est l'objet de son roman Tâdo, Tâdo, wéé ! ou « No more baby » (2012), où le lignage, les liens de cousinage marquent, entre autres, le parcours biographique collectif que l'auteure reconstitue sur fond des luttes politiques du militantisme indépendantiste, et des « échecs » de la modernité dans ce parcours. Ce retour à la Terre, à la nature, est aussi au cœur des solutions pensées à la crise de l'Anthropocène, sous la forme de la place de l'être humain à reconnaître dans l'ensemble du 
système de la Nature. Cette place n'est pas celle du « superhumain » créé par le discours moderne, et qui aura conduit à l'irresponsabilité de l'humain devant ses propres conditions de vie sur la planète. De reconnaître cette «vulnérabilité », cette «fragilité » épistémique invoquée alors, ne signifie pas sa faiblesse, mais bien sa capacité à « composer » avec la Nature. C'est de là qu'il récupère sa force, c'est-à-dire sa profonde dimension ontologique qui ne peut se comprendre pardelà cette Nature.

\section{La question de l'Anthropocène}

Pour Glowczewski et Laurens, qui en font la matière de leur contribution au travail collectif consacré à la réflexion épistémologique sur l'ère déclarée de l'Anthropocène, ou de la crise de la modernité, la solution, l'une des solutions à cette crise, passe en effet par la réactivation de la vulnérabilité épistémique de l'être humain face au système de la nature. Il faut rappeler, pour le propos, que ce rapport à la vulnérabilité invoquée est au cœur du principe religieux, celui des «croyances » dans les sociétés traditionnelles (appelées primitives par le discours moderne), et qui correspond au principe animiste. Ce principe établit un rapport différent entre l'être humain et la Nature, un rapport qui, sur le plan épistémologique, ne peut correspondre au rapport de domination de cette Nature tel que le conçoit le discours moderne qui aura conduit à la crise écologique et multisectorielle qui est évoquée désormais. Ce principe animiste est foncièrement au cœur de l'œuvre de la romancière guadeloupéenne Simone Schwarz-Bart, dont il peut être souligné ici la portée également foncièrement autonomiste ou indépendantiste. C'est une œuvre marquée par le discours identitaire antillaniste, certes, mais qui pose aussi la Guadeloupe comme « pays » à construire, et qui met en écriture, comme partie intégrante de cette construction, l'ordre du surnaturel. À ce titre, son œuvre et son discours identitaire tomberaient sous la catégorie du nativisme.

Pour Glowczewski et Laurens, dans leur article au titre significatif, «Le conflit des existences à l'épreuve du climat, ou l'Anthropocène revu par ceux que l'on préfère mettre à la rue ou au musée ", l'histoire de l'arrogance de la modernité s'exprime jusque dans sa tentative de conceptualisation de la crise qu'elle aura générée sur la planète, en inscrivant l'être humain en agentivité dans cette crise, notamment dans le paradigme de l'Anthropocène et son préfixe anthropos, c'est-à-dire humain. Nous reprenons en extension leur réflexion : 
À bien des égards l'Anthropocène semble être la nouvelle modalité sémantique du projet humaniste qui visait à nier dans l'universalisme la diversité des sociétés humaines. Initié par l'Homo industrialis, l'humanisme supposait à toutes les sociétés le même but: atteindre le confort et le style de vie de l'Europe colonisatrice. Il faisait ainsi de cette petite implantation humaine continentale, l'Europe, le destin de toute l'humanité, justifiant la soumission ou l'extermination des autres peuples, animaux et territoires. Avec l'Anthropocène, l'Homo industrialis techno-mondialisé, continue à nier la variété des aspirations humaines, des plus prosaïques aux plus poétiques, ainsi que la diversité des possibilités d'organisation sociales et politiques de nos vies, en disant une nouvelle fois que l'ensemble de l'humanité serait tendue vers une même destinée : devenir une force géologique. La fascination pour la puissance et la transformation matérielle de la terre n'a pas changé, le rêve consiste toujours à s'émanciper du ciel.

Pourtant, de plus en plus de chercheurs et d'activistes font l'apologie de la fragilité, comme Geneviève Azam (2015a) qui, dans son livre Osons rester humain, critique la fiction surpuissante de l'homme « augmenté » dans un monde adapté.

«Certains voient dans le changement climatique une opportunité technique et économique, susceptible de permettre un nouvel élan. Loin d'accepter la fragilité des écosystèmes et des sociétés, ils y voient un manque à combler, une erreur de la nature, un défaut de rationalité. Les solutions préconisées vont alors dans le sens d'une voloné de maîtrise redoublée, appuyée sur l'utilisation de techniques dites vertes - certaines visant à adapter les humains et la planète en les reconfigurant. » (Azam 2015b). Pour nous, la solution n'est pas de reconfigurer les humains et la planète en les adaptant mais, à l'instar d'Azam et de nombreux mouvements dans le monde, de « cultiver la fragilité au lieu de la vaincre et [de] rétablir avec la nature des liens de coopération » (Azam 2015a). (142-143)

Leur objectif est ainsi d'opposer à cette surévaluation de l'être humain, et particulièrement de celui de l'Occident, la vulnérabilité épistémique de l'humain comme espèce, de le « rasseoir » dans son espace naturel. En cela, il n'est plus, justement, habitant, mais habité par la terre, dans tout ce que cela peut signifier d'ontologique, d'humilité, et de respect de cette Nature pour sa propre survie :

Le moment nous semble aussi venu de renverser la fameuse formule poétique de Hölderlin pour dire, non plus que «l'homme habite poétiquement la terre » mais, à l'inverse, que la terre habite l'ensemble des êtres qui la peuplent $»^{1}$. Et que la terre, ou quelque chose comme l'esprit de la terre, nous habite tous, humains et nonhumains, animaux, pierres ou fleurs, de manière atmosphérique ; légère et fragile, mais aussi très concrète, à travers l'air qui relie l'ensemble des êtres. (Glowczewski et Laurens 143)

C'est en cela qu'une telle fragilité épistémique rencontre le principe animiste que retenait déjà Schwarz-Bart contre l'hégémonie assimilationniste dans sa Guadeloupe-Pays. Que ce soit dans son Ti Jean L'Horizon (1979), ou dans Pluie et vent sur Télumée Miracle (1972), la réalité donnée au personnage pour son symbolisme dans la question identitaire collective explorée ne se départ 
jamais de cette perspective animiste qui fait côtoyer le monde du surnaturel et anime la nature bien au-delà du simple décor narratif. Tel est le cas, ici, dans Pluie et vent sur Télumée Miracle, où le rêve du personnage s'écrit chez l'auteure dans les mêmes tournures du surnaturel qui se précisent dans l'autre roman, Ti Jean L'Horizon :

Cependant que je me parlais ainsi, nulle consolation ne me venait et j'ouvrais les yeux tout grands sur moi-même, et le soleil se couchait et la nuit tombait là-dessus, et le même soleil se levait le lendemain et je voyais maintenant qu'aucun fil ne reliait plus ma case aux autres cases. Alors je m'allongeais à même le sol et m'efforçais de dissoudre ma chair, je m'emplissais de bulles et tout à coup je me sentais légère, une jambe m'abandonnait puis un bras, ma tête et mon corps entier se dissipaient dans l'air et je planais, je survolais Fond-Zombi de si haut qu'il ne m'apparaissait plus que comme un grain de pollen dans l'espace. Mais j'atteignais rarement un tel bonheur et c'est à peine si je parvenais à contempler avec sérénité ma vie pillée, à peine si elle évoluait devant mes yeux à la manière d'un rêve anodin et important, mystère douloureux qui m'étonnait, m'échappait... (Pluie et vent, 157-158)

Dans Ti Jean L'Horizon, en effet, la métamorphose du personnage participe de la logique du récit et de son intelligibilité discursive et identitaire. Le constat du retour du temps colonial et sa réprobation, par exemple, qui forment bien écho avec le « retour» de la colonisation « réussie » de la Départementalisation, le sont dans la hauteur physique, éthique et symbolique que l'écriture confère au personnage tiré de la tradition orale, Ti Jean, et doté de pouvoirs surnaturels :

La trompe d'un navire résonna derrière le promontoire. Et revenant à l'heure présente, il songea que temps était venu maintenant de se couvrir de peau humaine, pour s'en aller au-devant de la nouvelle histoire qui l'attendait, la plus abracadabrante et saugrenue de toutes, en couillonnade, car elle n'était même plus la sienne. Indécis, il sautilla vers le buisson où avait abandonné le mousquet, le ceinturon et l'anneau de Connaissance. Mais une nouvelle vague de rire le prit, comme il en approchait, et l'idée lui vint d'une petite virée de jeune homme par-dessus la Guadeloupe, afin de voir ce que le pays était devenu depuis la dernière fois. Une patte en l'air, il se demanda un instant comment démêler son vouloir ; puis, soulevé par le rire, franc.

Sur la route coloniale, un groupe de négresses se dirigeaient vers la Pointe-à-Pitre, à la queue leu leu, chacune surmontée d'un lourd plateau de fruits et légumes. [...] Le cœur étreint d'une crainte obscure, qui lui rappelait celle qu'il avait connue dans le canot, à observer le jeu mystérieux des maîtres et des esclaves, Ti Jean survola successivement un âne, un attelage de bœufs, une troupe d'esclaves qui se rendaient au travail, la houe sur l'épaule, apparemment libres comme l'air. Sitôt après, il eut la vision d'un véritable carrosse d'antan, une voiture dorée comme dans les livres d'images et qui cahotait sur la route, conduite par un vieux cocher noir en livrée et haut-de-forme. Deux esclaves luisants de sueur couraient audevant des chevaux, un fanal allumé à bout de bras, et un vertige le saisit à ce 
spectacle : serait-il pas tombé dans un autre temps, une autre Guadeloupe, de même qu'il avait chu autrefois dans une Afrique oubliée, révolue... ? (282-283)

Pour les deux critiques de l'Anthropocène, la Nature est une force dont l'agentivité expliciterait la crise de l'Anthropocène, moins que l'action humaine, tel qu'initialement posé. Moins que l'être humain qui « détruit » la planète, c'est bien alors la planète qui « réagit » à l'action humaine, dans ce qui devient la crise existentielle de l'espèce. La crise du coronavirus du début de l'année 2020 forme écho avec une telle réflexion qui date d'aussi proche que l'année 2018, où 1'air de la planète entre-temps est devenu le porteur de la maladie qui tue l'être humain. Les deux critiques reviennent sur cette dimension animiste à retrouver de la Nature et du rapport de l'être humain à cette Nature :

Dans son livre Comment la terre s'est tue (2013), David Abram en appelle à la magie des sens pour retrouver les relations entre l'air, la terre et les vivants. Comme lui, nous pensons que « la terre s'est tue », mais seulement pour ceux qui ne savent pas l'écouter. Certains l'entendent encore et n'arrêtent pas de nous le rappeler alors qu'on préfère les mettre au musée, ou à la rue. Ce sont les peuples, réfugiés de la misère provoquée par leur colonisation et la société marchande, avide de saccager leurs terres pour s'approprier toujours plus de ressources. Si la terre s'est tue pour ceux qui l'exploitent sans retenue, de même l'écriture s'est tue pour un grand nombre de chercheurs des sciences dures ou dites humaines ou sociales, qui n'en finissent pas «d'apprendre à s'adapter», injonction de l'emprise néolibérale dénoncée par Pignarre et Stengers dans la Sorcellerie capitaliste (2013). Krishnamurti disait non sans humour que ce n'était pas forcément un signe de bonne santé mentale que d'être adapté à une société malade. (Glowczewski et Laurens 144-145)

C'est ce nativisme, aux antipodes alors de la pensée rationaliste, économiste, anti-humaine et capitaliste, qu'il est nécessaire de lire chez les écrivains, écrivaines qui en font la matière de leurs écritures, de leur refus d'un ordre mondial anti-humain, de leur évocation de cet autre monde que le discours moderniste qualifie d'utopique et que la romancière Gorodé, qui écrit dans les années 2000, restitue dans son roman-actualité. Dans son Tâdo, Tâdo, wéé! ou «No more baby », c'est ainsi tout le vingtième siècle du mouvement politique pour l'indépendance de la NouvelleCalédonie qui est mis en scène, à travers la famille et la coutume, mais aussi la voix, quasiautobiographique, de la femme kanak profondément ancrée dans la Terre, la terre kanak, la terre ancestrale de l'igname, que le lecteur découvre en même temps que la « sanction », pour un monde meilleur, que formule la romancière contre l'ordre colonial et l'ordre impérialiste qui menace la vie sur la planète : 


\section{DEBOUT}

Au bord du millénaire / se tient Âboro / l'être en tout / ce qu'il est / en tout lieu / en tout temps / à l'orée du sanctuaire / se tient Âboro / l'être pin colonnaire / l'homme araucaria / l'être cocotier / la femme taro d'eau / en lisière d'avenir / se tient Âboro / l'homme debout / en api tuteur d'igname / l'être tutélaire / la femme obsidienne / perle de rosée lunaire / en pluie diluvienne / aux frontières du futur $[\ldots]$

Dorez-vous longtemps au soleil / dans la paix du cœur et de l'être / et que cette histoire vous endorme / ou qu'elle vous réveille / trouvez-lui une suite / en prenant votre temps au soleil / Cîgadoo. (348-350)

Le nativisme n'est donc pas le passé que cherche à faire revivre le sujet colonisé ou postcolonial, dans bien des cas, mais le quotidien de collectivités dont le discours moderniste ne reconnaît pas la pertinence ou simplement l'existence. La théorie postcoloniale est en mesure désormais de sortir des catégories qu'imposait la réalité planétaire des années 1980 et 1990 de son émergence, la réalité de la mondialisation économiste et occidentale, avec le triomphe achevé de l'État moderne et de ses appareils de maintien. Elle est amenée, fort de son engagement herméneutique, mais aussi éthique, à participer à la reformulation des termes de la crise de l'Occident dans la question du «proto-Anthropocène », pour lire ce nativisme du quotidien par lequel l'écrivain, l'écrivaine francophone jugulait déjà les conséquences de l'histoire moderne, de l'histoire coloniale, une histoire que nous appelons aujourd'hui « crise de l'Anthropocène ». Je voudrais terminer le propos sur les mots des deux critiques, Glowczewski et Laurens, qui en appellent à la responsabilité du travail herméneutique - critique - dans le retour nécessaire à la Terre, à la Mère-Nature, tel que le proposait aussi la perspective écolinguistique : «Ne serait-ce pas notre tâche comme enseignants ou chercheurs, nous qui essayons, que ce soit en anthropologie ou en architecture, de dessiner des existences dans leur milieu vivant, de trouver les mots pour animer la terre, plutôt que d'alimenter les débats qui n'en finissent pas de diagnostiquer sa mort?» (145).

\section{Bibliographie}

Abram, David. The Spell of the Sensuous. New York : Pantheon, 1990.

--- . Comment la terre s'est tue. Pour une écologie des sens. Paris : La Découverte, 2013.

Azam, Geneviève. Osons rester humain. Les impasses de la toute-puissance. Paris : Les Liens qui libèrent, 2015. 
---. « Mobilisation civile contre le « crime climatique ». La destruction des fondements vitaux de l'existence n'est pas une fatalité ». La Croix. 4 septembre 2015.

Beau, Rémi et Catherine Larrère. Penser l'Anthropocène. Paris : Les Presses SciencesPo, 2018.

Diawara, Manthia. « The Nature of Mother in Dreaming rivers ». Third Text 13 (1990-91) : 7384.

Harris, Wilson. History, Fable and Myth in the Caribbean and Guianas. Georgetown, Guyana: The National History and Arts Council, Ministry of Information and Culture, 1974.

Glissant, Edouard. Caribbean Discourse : Selected Essays. Tr. Michael Dash. Virginia : UP of Virginia, 1989.

Glowczewski, Barbara et Christophe Laurens. «Le conflit des existences à l'épreuve du climat, ou l'Anthropocène revu par ceux que l'on préfère mettre à la rue ou au musée ». Beau et Larrère. 141-155

Gorodé, Déwé. Tâdo, Tâdo, wéé ! ou «No more baby». Pirae : Au Vent des îles, 2012.

Grosz, Elizabeth. « Judaism and Exile : The Ethics of Otherness ». New formations 12 (1990) : 7788.

Parry, Benita. «Resistance Theory / Theorising Resistance or Two Cheers for Nativism ». Colonial Discourse, Postcolonial Theory. Dir. F. Barker, P. Hulme et M. Iversen. Manchester : Manchester UP, 1994.

Schwarz-Bart, Simone. Pluie et vent sur Télumée-Miracle. Paris : Seuil, rééd. coll. « Points », 1995 (1972).

---. Ti-Jean L'Horizon. Paris : Seuil, rééd. coll. « Points », 1998 (1979).

Stengers, Isabelle. Au temps des catastrophes. Résister à la barbarie qui vient. Paris : La Découverte, 2013 ( 2009).

Stibbe, Aran. Ecolinguistics. Langage, Ecology and the Story We Live by. Frankfort : Routledge, 2015.

\section{Note}

1. Atelier de Christophe Laurens au séminaire Humanisme et mindfulness (pleine présence) au centre bouddhiste du domaine d'Avalon, 2015 (Glowczewski et Laurens 143, note 7). 